\title{
Design of piezoelectric transducers using topology optimization
}

\section{Emílio Carlos Nelli Silva $\nmid$ and Noboru Kikuchił}

Department of Mechanical Engineering and Applied Mechanics, The University of Michigan, Ann Arbor, 3005 EECS, MI 48109-2125, USA

Received 8 October 1997

\begin{abstract}
Among other applications piezoelectric transducers are widely used for acoustic wave generation and as resonators. These applications require goals in the transducer design such as high electromechanical energy conversion for a certain transducer vibration mode, specified resonance frequencies and narrowband or broadband response. In this work, we have proposed a method for designing piezoelectric transducers that tries to obtain these characteristics, based upon topology optimization techniques and the finite element method (FEM). This method consists of finding the distribution of the material and void phases in the design domain that optimizes a defined objective function. The optimized solution is obtained using sequential linear programming (SLP). Considering acoustic wave generation and resonator applications, three kinds of objective function were defined: maximize the energy conversion for a specific mode or a set of modes; design a transducer with specified frequencies and design a transducer with narrowband or broadband response. Although only two-dimensional plane strain transducer topologies have been considered to illustrate the implementation of the method, it can be extended to three-dimensional topologies.

Transducer designs were obtained that conform to the desired design requirements and have better performance characteristics than other common designs.
\end{abstract}

\section{Introduction}

Piezoelectric transducers have the capability of converting mechanical energy into electrical energy and vice versa. They have been used extensively in acoustic wave generation devices such as ultrasonic transducers, and as resonators in measuring instruments, microprocessors and various kinds of electronic equipment.

Depending on the application, there are different goals for transducer design. For most acoustic wave generation applications, the transducer is required to oscillate in the 'piston' mode (ideal for generation of acoustic waves) [1]. In addition, the transducer must be designed to have a broadband or narrowband frequency response which defines the kind of acoustic wave pulse generated (short pulse or continuous wave, respectively). In the case of resonator design, the requirements include (1) an assurance of the specified mechanical resonance frequency, (2) an absence of 'spurious' resonances close to the working frequency and (3) a high quality factor $\left(Q_{m}\right)$ including minimum energy dissipation in the material and in the attachments. The first requirement is critical since very small deviations in the resonance frequency may disable the electronic equipment. Regarding the second requirement, the number of unwanted resonances and their proximity to the working frequency determine the performance of the resonator in terms of its frequency

\footnotetext{
$\dagger$ Assistant Professor, Department of Mechanical Engineering of Polytechnic School at the University of São Paulo, São Paulo, Brazil. E-mail address: ecnsilva@usp.br.

$\ddagger$ Professor.
}

response. The design of single-frequency resonators is one of the most important problems in piezoelectric engineering [2].

These goals of transducer design are essentially related to the transducer resonance frequency, vibration modes and the electromechanical coupling factor (EMCC), which measures how strong the excitation of a specific transducer mode is in the transducer response. All these parameters depend on many factors, the most important being the transducer topology (or shape) and material properties. By changing the transducer topology we can try to achieve the abovementioned goals.

In this sense, some previous papers have reported the study of the dependence of the resonance frequency and the EMCC on the dimension ratio of simple shapes of piezoelectric transducers (such as cubic and cylindrical). Using the finite element method (FEM), Kunkel et al [1] built dispersion curves of resonance and antiresonance frequencies and EMCC as a function of diameter/thickness ratio for cylindrical transducers. These curves helped our understanding of the different kinds of transducer vibration mode, as well as the change in the EMCC for each mode with the transducer dimension ratio. Other authors, such as Challande [3] and Sato et al [4], discussed the optimization of the dimension ratio of a piezoelectric element with parallelepipedic shape that maximizes the EMCC for a specific mode. The piezoelectric elements were embedded as inclusions in the polymer matrix to build piezocomposite transducers. No optimization method was used and the 
optimal ratio was obtained by building curves of the objective functions (resonance frequency or EMCC) as a function of the design variables (dimension ratios). The shape was fixed and only the dimension ratios were changed, thereby limiting the problem to a sizing optimization. To our knowledge, the first work applying an optimization method for piezoelectric transducer design was developed by Simson and Taranukha [2] who optimized the thickness distribution of a piezoelectric resonator to detune the working frequency from spurious anharmonics. The problem consisted of a sizing optimization since only the thickness distribution was optimized. Their formulation is based on an eigenvalue optimization problem that is solved by using mathematical programming integrated with the finite element method. However, the piezoelectric effect was not taken into account in their FEM formulation.

In this work, we intend to define the first steps for designing piezoelectric transducers and resonators by applying topology optimization techniques. Considering the discussion presented before, the problem of designing a piezoelectric transducer or resonator is posed as an eigenvalue optimization problem in structural optimization. The topology of the transducer is obtained by searching for the optimal distribution of two phases (material and void) in the design domain, assuming that the properties can vary from one phase to another proportionally to a fraction parameter $x$ which is the design variable in the problem. Since complex topologies are expected, the finite element method is used for transducer modeling.

Although the method introduced in this work is general and can be applied in the design of three-dimensional (3D) transducers, the examples presented herein are limited to two-dimensional (2D) plane strain models due to lower computational cost. The plane stress condition could also be considered, but it is less realistic (due to manufacturing considerations) than the plane strain condition for representing the transducer operation. The transducers are considered to be surrounded by air, and no damping effect is considered in the modeling.

This paper is organized as follows: in section 2, we present a short introduction to piezoelectric transducer modeling using FEM, and we define quantities such as resonance and antiresonance frequencies and the electromechanical coupling coefficient (EMCC). These quantities describe the piezoelectric transducer behavior. In section 3, the optimization problem and its parameters are defined. In section 4 , transducer topologies resulting from the optimization are presented and the results are discussed. In section 5, some conclusions are given. The symmetry conditions considered for the 2D plane strain FEM model are briefly presented in appendix A, and the sensitivity analysis of the transducer eigenvalues necessary for the optimization problem is derived in appendix B.

\section{Piezoelectric transducer modeling}

Since we expect topology optimization to result in complex topologies, a general method such as the finite element method is necessary for the structural analysis. Therefore, in this section we describe briefly the finite element formulation applied to piezoelectricity. We also define some important quantities such as the resonance and antiresonance frequencies and the electromechanical coupling factor (EMCC). These quantities characterize the behavior of the piezoelectric transducer.

\subsection{FEM applied to piezoelectricity}

The finite element equations for modeling a linear piezoelectric medium were developed by [5], [6]. Considering time harmonic excitation, these equations may be written as

$$
\left(-\omega^{2}\left[\begin{array}{cc}
\mathbf{M} & \mathbf{0} \\
\mathbf{0} & \mathbf{0}
\end{array}\right]+\left[\begin{array}{cc}
\boldsymbol{K}_{u u} & \boldsymbol{K}_{u \phi} \\
\boldsymbol{K}_{u \phi}^{T} & -\boldsymbol{K}_{\phi \phi}
\end{array}\right]\right)\left\{\begin{array}{l}
\boldsymbol{U} \\
\boldsymbol{\Phi}
\end{array}\right\}=\left\{\begin{array}{l}
\boldsymbol{F} \\
\boldsymbol{Q}
\end{array}\right\}
$$

where $\mathbf{M}, \boldsymbol{K}_{\boldsymbol{u} u}, \boldsymbol{K}_{\boldsymbol{u} \phi}$ and $\boldsymbol{K}_{\phi \phi}$ are the mass, stiffness, piezoelectric and dielectric matrices, respectively, and $\boldsymbol{F}, \boldsymbol{Q}$, $\boldsymbol{U}$ and $\boldsymbol{\Phi}$ are the nodal mechanical force, nodal electrical charge, nodal displacements and nodal electric potential vectors, respectively. Since the piezoelectric medium is considered to be an insulator, $Q$ has non-zero terms only in the electrodes [5]. This system of equations describes the FEM model for piezoelectricity without considering the damping effect, and assumes that the piezoelectric structure is free in air, that is, it is not coupled to any propagation medium. For more details, the reader should refer to [5], [6].

\subsection{Characteristic frequencies}

The two resonance frequencies that characterize the behavior of the piezoelectric transducer for different vibration modes are the resonance $\left(\omega_{r}\right)$ and antiresonance $\left(\omega_{a}\right)$ frequencies. Resonance $\omega_{r}$ is the piezoelectric resonance for an excitation by electric potential, and antiresonance $\omega_{a}$ is the piezoelectric resonance for an excitation by electric charge. In fact, these frequencies correspond to the antiresonance and resonance of the transducer electric impedance, defined as the ratio between the voltage and electrical current in the transducer electrodes $[5,6]$.

Using FEM, these frequencies can be obtained by solving two eigenvalue problems generated by two different electrical boundary conditions in equation (1), considering $\boldsymbol{F}=\mathbf{0}$ [7]. For the resonance frequency we consider that the electrodes are short-circuited, that is, the electrical potential degrees of freedom (DOFs) in both electrodes are set to zero $\left(\Phi_{e}=\mathbf{0}\right)$ in equation (1) (potential boundary conditions). For the antiresonance frequency $\left(\omega_{a}\right)$ the transducer is an open circuit, that is, the electrical charges are set to zero in the electrodes $(\boldsymbol{Q}=\mathbf{0})$. In the FEM model, considering that one electrode is always grounded $\left(\boldsymbol{\Phi}_{e}=\mathbf{0}\right)$, the two eigenvalue problems can be obtained simply by changing the electrical boundary conditions in the other electrode, that is, the electrical potential DOFs are set to zero $\left(\boldsymbol{\Phi}_{e}=\mathbf{0}\right)$ for frequency $\omega_{r}$, and for frequency $\omega_{a}$ they are constrained to be equipotential and we consider $\boldsymbol{Q}=\mathbf{0}[7,5]$.

There are many ways of solving these eigenvalue problems. The most direct way is just to impose in equation (1) the boundary conditions discussed and solve each of the eigenvalue problems. The advantage of this method is that the band of the 'total stiffness' matrix, involving elastic, piezoelectric and dielectric terms, 
is preserved reducing the storage space requirements. However, we have to deal with a mass matrix that is positive semi-definite (due to the zero mass terms related to the electrical degrees of freedom), and the 'total stiffness' matrix is indefinite (negative and positive eigenvalues) due to the negative diagonal terms of the dielectric submatrix. As a consequence, a direct solution using traditional eigenvalue solvers such as the subspace iteration method does not work and an alternative method must be used. A solution for such a case is proposed in [7], [8] and [9].

Another method for solving the eigenvalue problems is to apply a static condensation to the system (1) to eliminate the internal electrical potential DOFs (since the internal electrical nodal charges are zero), keeping in the formulation only electrical potential DOFs of the electrodes, as described in [5]. The advantage of this method is that the matrices obtained are positive definite, and therefore, the eigenvalue problem can be solved by directly applying the usual solvers. However, the band of the 'total stiffness' matrix is destroyed and we must deal with a full matrix which increases the memory requirements consisting of a high drawback if we have to deal with large FEM models.

In this work, since the optimization method itself has memory requirements, and in topology optimization we usually need to deal with large models to try to get a clear image of the final topology, the first approach proposed by [8] was implemented using the program DNLASO by D S Scott from the LASO package (available through NETLIB) which is based on the block Lanczos algorithm.

\subsection{EMCC}

The electromechanical coupling coefficient (EMCC or $k$ ) is defined for each vibration mode and measures the strength of the mode's excitation, that is, how strong the couple is between the mode and the excitation. The most general definition of $k$ is given by the expression:

$$
k^{2}=\frac{E_{e m}^{2}}{E_{m} E_{e}}=\frac{\left(\boldsymbol{U}^{T} \boldsymbol{K}_{u \phi} \boldsymbol{\Phi}\right)^{2}}{\left(\boldsymbol{\Phi}^{T} \boldsymbol{K}_{\phi \phi} \boldsymbol{\Phi}\right)\left(\boldsymbol{U}^{T} \boldsymbol{K}_{u u} \boldsymbol{U}\right)}
$$

where $E_{e m}, E_{e}$ and $E_{m}$ are the electromechanical, elastic and electrical energy, respectively, defined in [5] and [6]. $\boldsymbol{U}, \boldsymbol{\Phi}$, $\boldsymbol{K}_{u \phi}, \boldsymbol{K}_{\phi \phi}$ and $\boldsymbol{K}_{u u}$ were defined above.

However, Naillon et al [5] showed that this expression can be approximated by an equation involving the frequencies $\omega_{r}$ and $\omega_{a}$ (defined above):

$$
k^{2} \cong \frac{\omega_{a}^{2}-\omega_{r}^{2}}{\omega_{r}^{2}}=\frac{\lambda_{a}-\lambda_{r}}{\lambda_{r}}
$$

and

$$
\lambda_{a}=\omega_{a}^{2} \quad \lambda_{r}=\omega_{r}^{2}
$$

where $\lambda_{r}$ and $\lambda_{a}$ are the resonance and antiresonance eigenvalues, respectively.

Through the EMCCs for the different modes it is possible to know which modes will predominate in the excitation. If we want to increase the contribution of a specific mode we must increase its $k$ [6]. Therefore, if we desire a narrowband transducer we must design a piezoelectric structure with an operational frequency that is not only far from the other frequencies but that also has a large $k$, and the other modes must have a low $k$. For a multimode broadband transducer we must have the resonance frequencies in the desired band close to each other and with high $k$, and the other modes must have a low $k$.

\section{Topology optimization}

In this section, the procedure and numerical implementation of topology optimization for designing piezoelectric transducers is described. The objective functions, constraints and material model applied are discussed.

\subsection{Problem formulation}

In this work, the problem of designing piezoelectric transducers was posed essentially as an eigenvalue optimization problem similar to the one described in $[10,11]$. The main problem in the eigenvalue optimization is the 'switching' of the vibration modes during the optimization, which makes it difficult to control the maximization (or minimization) of the objective function if this is defined only as a function of one eigenvalue. In order to overcome this switching, Ma et al [10] proposed multiobjective functions written in terms of many eigenvalues. Therefore, even though the modes switch during the optimization, the value of the multiobjective function involving many eigenvalues is maximized or minimized. Considering the typical goals in transducer design described in the introduction, three kinds of objective function are defined.

The first one consists of the maximization of the EMCC ( $k$ ) for a specific mode or set of modes. It is a multiobjective function that is defined following the concept described in [10], and is given by the equation:

$$
F_{1}=\alpha\left(\sum_{i=1}^{m} \frac{w_{i}}{k_{i}^{2}}\right)^{-1} \text { and } \alpha=\sum_{i=1}^{m} w_{i}
$$

where $w_{i}$ is the weight coefficient, $k_{i}$ is the EMCC for mode $i$ $(i=1,2, \ldots, m)$ and $m$ is the number of modes considered in the multiobjective function.

The second objective function is related to the design of a transducer with specified resonance frequencies. It is also a multiobjective function defined in [10], and given by the equation:

$$
F_{1}=\left(\frac{1}{\alpha} \sum_{i=1}^{m} \frac{1}{\lambda_{o_{i}}^{2}}\left(\lambda_{i}-\lambda_{o_{i}}\right)^{2}\right)^{1 / 2} \text { and } \alpha=\sum_{i=1}^{m} \frac{1}{\lambda_{o_{i}}^{2}}
$$

where $\lambda_{i}$ is the eigenvalue of order $i(i=1,2, \ldots, m), \lambda_{o_{i}}$ is the specified eigenvalue for mode $i(i=1,2, \ldots, m)$ and $m$ is the number of modes considered in the multiobjective function. This problem is difficult to solve even for mechanical structures (no piezoelectric effect). However, it can be used, for example, to design a piezoelectric transducer with undesired frequencies tuned out from the main operational frequency, since in this case we do not need to achieve the specified frequency values, but only make those frequencies far from the operational frequency.

Finally, the third objective function is related to the design of narrowband or broadband transducers. A 
narrowband transducer presents one mode with a strong contribution to the transducer response, and the other modes must have resonance frequencies far from the resonance frequency of this strong mode with a weak contribution to the transducer response. This characterizes a unimodal behavior in the transducer response. For a broadband transducer, a possible design could be to keep the resonances in the desired frequency band close to each other, and with their respective modes making a strong contribution to the transducer response. The other modes (out of the desired band) must have a weak contribution to the transducer response. This characterizes not only a broadband but also a plurimodal (or multimode) behavior in the transducer response (many modes have strong contribution to the response). An application would be the design of a multimode broadband Tonpilz transducer as described in [12]. This type of transducer is used in high accuracy and high resolution sonar systems that require broadband transducers. Therefore, as a first step in the design of a narrowband and broadband transducer, the third objective function is a multiobjective function defined as a linear combination of the previous ones. It is given by the equation:

$$
\begin{aligned}
F_{3} & =\beta * \bar{F}_{1}-(1-\beta) * \bar{F}_{2} \\
0 & <\beta<1
\end{aligned}
$$

where $\beta$ is a weight coefficient, and $\bar{F}_{1}, \bar{F}_{2}$ are scaled values of $F_{1}$ and $F_{2}$, respectively. The minus sign is required because the function $F_{2}$ must be minimized. The objective is to control not only the distance between the frequencies but also how strongly their modes contribute to the transducer response (EMCC of each mode).

Considering all these features, the final optimization problem can be stated as:

maximize: $F(\boldsymbol{x})$, where $\boldsymbol{x}=\left[x_{1}, x_{2}, \ldots, x_{n}, \ldots, x_{N D V}\right]$

$\boldsymbol{x}$

$$
\begin{gathered}
\text { subject to: }\left(\overline{\mathbf{K}}_{r}-\lambda_{r} \overline{\mathbf{M}}_{r}\right) \boldsymbol{W}_{r}=\mathbf{0} ; \text { and } \boldsymbol{W}_{r}=\left\{\begin{array}{c}
\boldsymbol{U}_{r} \\
\boldsymbol{\Phi}_{r}
\end{array}\right\} \\
\lambda_{r}=\omega_{r}^{2} \\
\left(\overline{\mathbf{K}}_{a}-\lambda_{a} \overline{\mathbf{M}}_{a}\right) \boldsymbol{W}_{a}=\mathbf{0} ; \text { and } \boldsymbol{W}_{a}=\left\{\begin{array}{c}
\boldsymbol{U}_{a} \\
\boldsymbol{\Phi}_{a}
\end{array}\right\} \\
\lambda_{a}=\omega_{a}^{2} \\
W>W_{\min } \\
0<x_{\min } \leqslant x_{n} \leqslant 1 \\
\text { symmetry conditions }
\end{gathered}
$$

where $\overline{\mathbf{K}}_{r}, \overline{\mathbf{M}}_{r}$ and $\overline{\mathbf{K}}_{a}, \overline{\mathbf{M}}_{a}$ are the 'total' stiffness and mass matrices (involving electrical and mechanical degrees of freedom) of the resonance and antiresonance problems, respectively. $\lambda_{r}, \lambda_{a}$ and $\boldsymbol{W}_{r}, \boldsymbol{W}_{a}$ are the resonance and antiresonance eigenvalues and modes, respectively. $F(\boldsymbol{x})$ is one of the functions defined earlier. The first and third $\left(F_{1}\right.$ and $\left.F_{3}\right)$ will be maximized and the second $\left(F_{2}\right)$ will be minimized.

To reduce the amount of gray scale in the final design, we define a 'cost function' constraint described by the expression:

$$
W=\int_{\Omega} x^{p} \mathrm{~d} \Omega \cong \sum_{n=1}^{N} x_{n}^{p} V_{n}
$$

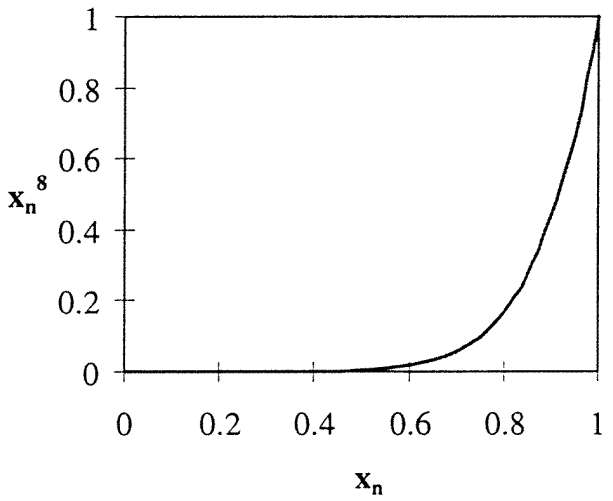

Figure 1. Cost function $W$ as a function of density $x_{n}$ in each finite element.

where $V_{n}$ is the volume of each finite element and $N$ the number of elements. A lower bound $W_{\min }$ is defined for this cost function. The value of the lower bound determines how efficiently the gray scale will be eliminated. A satisfactory value for $W_{\min }$ can be achieved by performing a certain number of trials (two or three) until we obtain a clear topology. The coefficient $p$ must be chosen to penalize the intermediate values of $x_{n}$. In this work, $p$ was chosen to be eight. Figure 1 shows the cost function as a function of the design variable $x_{n}$ for one finite element.

The value for $p$ was chosen taking the behavior of the objective function into account. Note that because of the chosen value for $p$, the lower and intermediate values of $x_{n}$ contribute almost equally in the cost function constraint. Therefore, if the maximization (or minimization) of the objective function tries to decrease $x_{n}$, intermediate values of $x_{n}$ will change for lower values. Larger values of $x_{n}$ will change to one to satisfy the lower bound constraint $W_{\min }$. This reduces the amount of gray scale in the solution. However, the cost of eliminating the gray scale is a reduction in the value of the objective function.

A lower bound $x_{\min }$ is also specified for design variables $x_{n}$ to avoid numerical problems (singularity of the stiffness matrix in the finite element formulation). In this work, $x_{\min }$ was chosen to be $10^{-4}$. Numerically, regions with $x_{n}=x_{\min }$ have practically no structural significance and can be considered void regions. Therefore, the bounds for the design variable are $0<x_{\min } \leqslant x_{n} \leqslant 1$.

Finally, we address the constraint related to the symmetry conditions. Since we are interested only in symmetric modes in relation to the $x$ axis and $y$ axis, only one quarter of the domain is considered. Symmetry reduces the computational cost. The symmetry conditions for displacements and electrical potential DOFs are expressed in the boundary conditions which are stated in appendix A.

\subsection{Material model for intermediate densities}

To define the material distribution, we need a material model that allows the properties in each element to assume intermediate values. In this work, the material properties in a given element are simply some fraction $x_{n}$ times the material properties of the basic material. This is an 'artificial' material model for intermediate densities, but since we obtain 
an entirely solid material or void in each element, this is a valid approach. However, during the optimization process intermediate densities are allowed and consist of artificial (non-existent) materials. By using this model the design procedure is greatly simplified [13, 14].

The basic material has a stiffness tensor $c_{i j k l}^{0}$, piezoelectric tensor $e_{i j k}^{0}$ and dielectric tensor $\epsilon_{i j}^{0}$. If the basic material considered in the analysis is steel (isotropic), the piezoelectric coefficients are zero, and the electric effect is not considered in the finite element. Therefore, the local tensor properties in each element $n$ can be expressed in terms of one design variable $x_{n}$ times the basic material property:

$$
c_{i j k l}^{n}=x_{n} c_{i j k l}^{0} \quad e_{i j k}^{n}=x_{n} e_{i j k}^{0} \quad \epsilon_{i j}^{n}=x_{n} \epsilon_{i j}^{0}
$$

where $x_{n}$ represents the amount of basic material in that element (local density) that ranges from $x_{\min }$ to 1 . For $x_{n}=x_{\min }$ the element is a 'void' and for $x_{n}=1$ the element assumes the properties of the solid material.

Considering that the design domain was discretized in $N$ finite elements, the material type changes from element to element during the optimization. The design problem consists of finding the fraction $x_{n}$ of material in each element such that the objective function is maximized or minimized.

\subsection{Implementation of the optimization procedure}

A flow chart of the optimization algorithm describing the steps involved is shown in figure 2. The software was implemented in FORTRAN. The initial domain is discretized by finite elements and the design variables are the values of $x_{n}$ (defined above) in each finite element.

In this work, we use the mathematical programming method called sequential linear programming (SLP). This method has been successfully applied to topology optimization (see [13], [14]) and in addition, the first author has previous experience with this method [15]. It consists of the sequential solution of approximate linear subproblems that can be defined by writing a Taylor series expansion for the objective and constraint functions around the current design point $x_{n}$ in each iteration step, as shown in equation (9) for the constraint function $W$.

$$
\begin{aligned}
W & =W\left(x_{n 0}\right)+\sum_{n \in S}\left(x_{n}-x_{n 0}\right) p x_{n 0}^{p-1} V_{n} \geqslant W_{\min } \\
& \Rightarrow \sum_{n \in S} x_{n} p x_{n 0}^{p-1} V_{n} \geqslant W_{\min }-W\left(x_{n 0}\right)+\sum_{n \in S} x_{n 0} p x_{n 0}^{p-1} V_{n}
\end{aligned}
$$

where $S$ is the set of elements considered in the design domain.

The linearization of the problem (Taylor series) in each iteration requires the sensitivities (gradients) of the objective function and constraints in relation to $x_{n}$. These sensitivities can be expressed as a function of the sensitivities of the resonance and antiresonance eigenvalues $\lambda_{r}$ and $\lambda_{a}$, respectively, derived in appendix B.

In each iteration, moving limits are defined for the design variables. Typically, during one iteration, the design variables will be allowed to change by $5-15 \%$ of their original values. After optimization, a new set of design variables $x_{n}$ is obtained and updated in the design domain. The linear

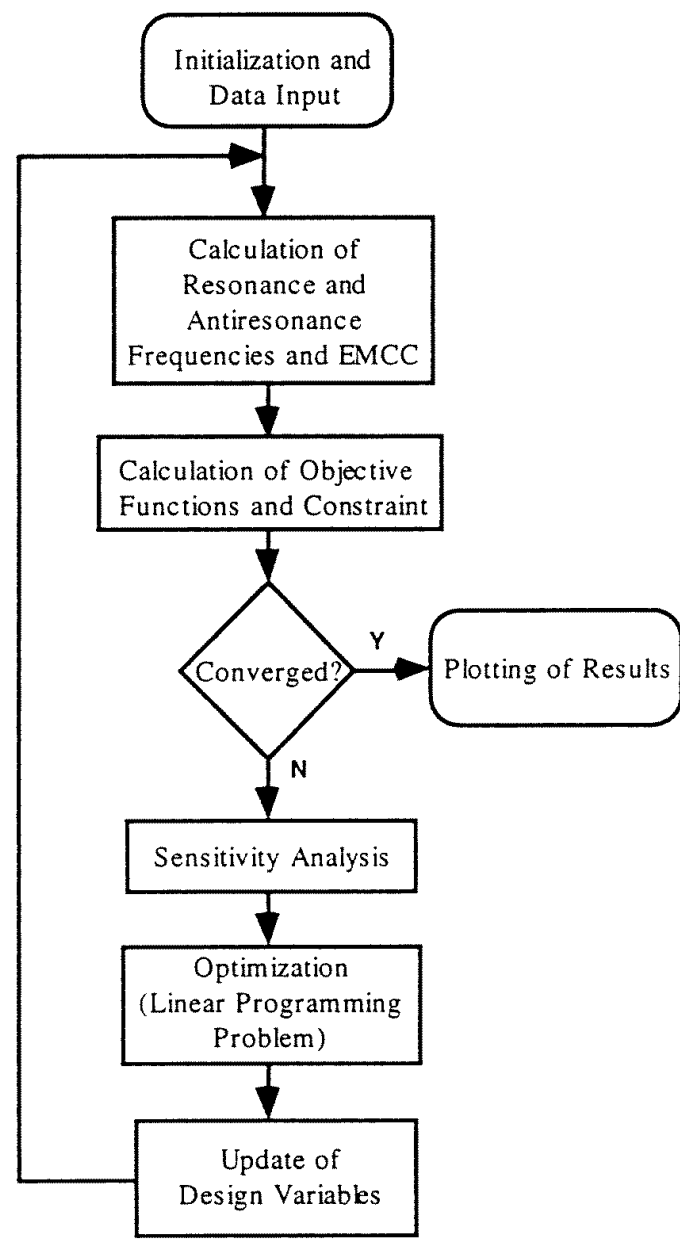

Figure 2. Flow chart of the optimization procedure.

programming subproblem in each iteration of the SLP is solved using the package DSPLP from the SLATEC library [16].

\section{Results}

In this section, we present results related to some typical goals in transducer design, such as maximizing the EMCC for a specific mode or a set of modes, designing a transducer with specified frequencies and designing a narrowband transducer and a broadband transducer. The values of EMCC obtained are compared with those for simple designs of transducers.

\subsection{Optimized piezoelectric transducers}

In this work, we consider 2D plane strain piezoelectric transducers. Since we are interested only in the symmetric modes of the transducer, the design was conducted in only one quarter of the domain by specifying the necessary boundary conditions as described in appendix A.

Before presenting the optimized transducers, the dispersion curves of the normalized resonance and antiresonance frequencies, and of EMCC (or $k$ ), for a 2D plane strain transducer of rectangular shape were built as a function of the domain dimension ratio (width/thickness). 


\section{Dispersion Diagrams of Resonance}

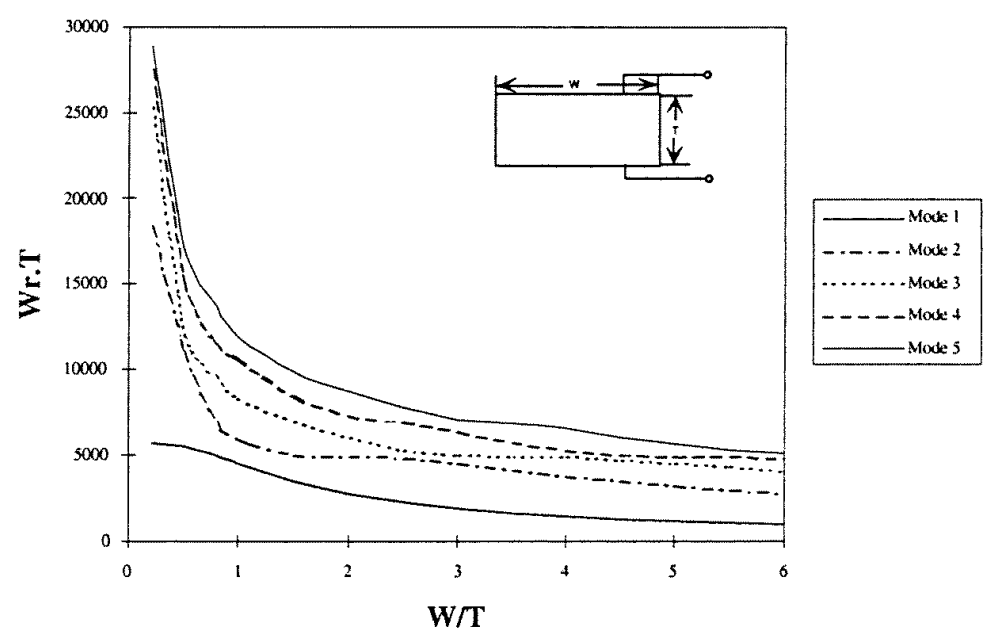

Dispersion Diagrams of Antiresonance

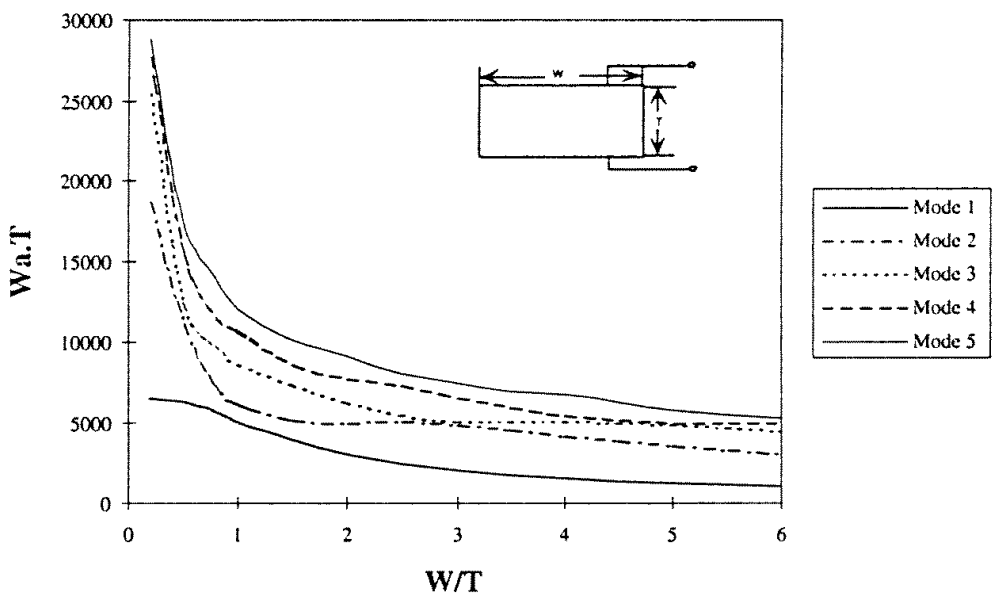

Figure 3. Dispersion curves of the normalized resonance $W_{r} T$ (cycles $\mathrm{m} \mathrm{s}^{-1}$ ) and antiresonance $W_{a} T$ (cycles $\mathrm{m} \mathrm{s}^{-1}$ ) for a $2 \mathrm{D}$ plane strain transducer of rectangular shape as a function of the domain dimension ratio width $(W) /$ thickness $(T)$. ( $T$ is the distance between the transducer electrodes.)

They are presented in figures 3 and 4 . These curves show the spectrum of normalized resonance and antiresonance frequencies that can be obtained as well as the maximum EMCC achieved by changing only the dimension ratio of the transducer with a simple rectangular shape. Therefore, they give us an idea of the characteristics achievable when only the dimension ratio is changed for a simple shape. The maximum values of EMCC for the simple rectangular shape will be compared with the values obtained for some optimized transducer topologies.

Piezoelectric materials used in transducer design are usually ceramics, and if they have complex shapes they are very difficult to fabricate. Therefore, two kinds of initial domain are considered. In the first model, there are two materials: steel and piezoceramic (PZT5A). The piezoceramic domain remains unchanged during the optimization. The steel is the design domain and thus the transducer will have a complex topology only in the steel domain. This model can be used, for example, in the design of the Tonpilz transducer [12]. This configuration was chosen to avoid the difficulty of manufacturing piezoceramics with complex topologies. Reasonable changes in the characteristic frequencies and EMCC (for different modes) of the transducer can be achieved by merely adding a steel coupling structure with complex topology to a simple shape PZT transducer. It was concluded that materials such as steel, aluminum and brass are better for this kind of design because they have a stiffness and density of the same order as piezoceramic, which allows large changes in the transducer frequencies with a small amount of material. Steel was chosen in this work. Figure 5 describes the relative dimensions of the initial domain considered. Of course, in practice, an insulator must be provided in the symmetry plane for the steel part, otherwise the electrodes will be short circuited. However, in the FEM model this effect does not occur since electrical degrees of freedom are considered only in the ceramic domain. In the second model, we consider changes in the ceramic topology. Therefore, the entire 


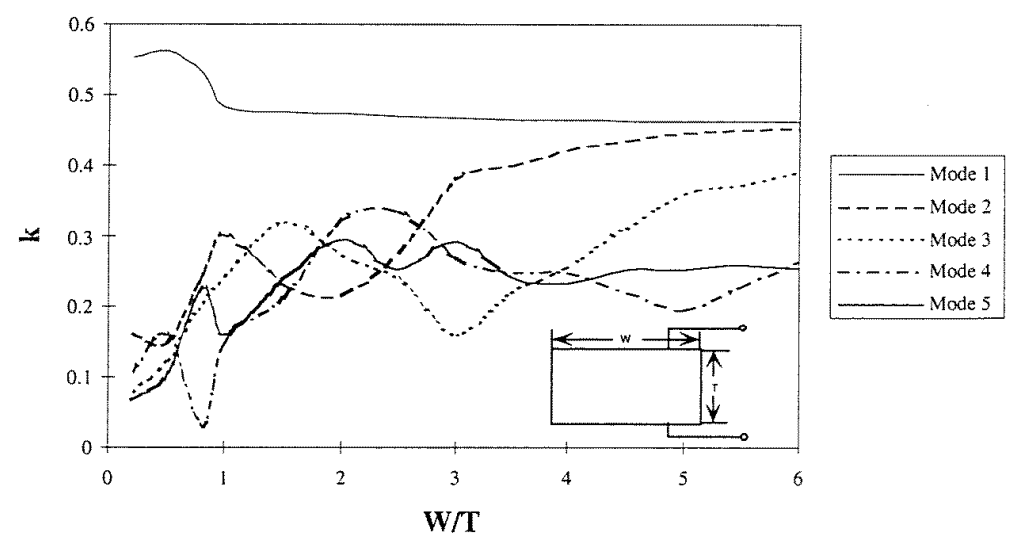

Figure 4. Dispersion curves of the EMCC $(k)$ for a 2D plane strain transducer of rectangular shape as a function of the domain dimension ratio width $(W) /$ thickness $(T)$.

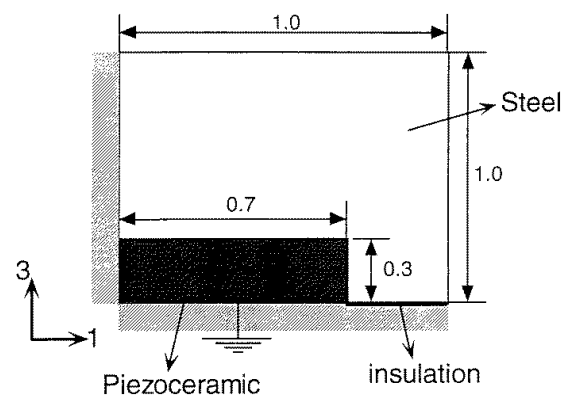

Figure 5. Configuration for the model PZT5A/steel with its relative dimensions. The piezoelectric domain remains unchanged. The piezoceramic is polarized in the 3 direction.

Table 1. Material properties of PZT5A and steel used in the simulations.

\begin{tabular}{lc}
\hline Piezoceramic PZT5A & \\
$c_{11}^{E}\left(10^{10} \mathrm{~N} \mathrm{~m}^{-2}\right)$ & 12.1 \\
$c_{12}^{E}\left(10^{10} \mathrm{~N} \mathrm{~m}^{-2}\right)$ & 7.54 \\
$c_{13}^{E}\left(10^{10} \mathrm{~N} \mathrm{~m}^{-2}\right)$ & 7.52 \\
$c_{33}^{E}\left(10^{10} \mathrm{~N} \mathrm{~m}^{-2}\right)$ & 11.1 \\
$c_{1212}^{E}\left(10^{10} \mathrm{~N} \mathrm{~m}^{-2}\right)$ & 2.10 \\
$c_{1313}^{E}\left(10^{10} \mathrm{~N} \mathrm{~m}^{-2}\right)$ & 2.30 \\
$e_{13}\left(\mathrm{C} \mathrm{m}^{-2}\right)$ & -5.4 \\
$e_{33}\left(\mathrm{C} \mathrm{m}^{-2}\right)$ & 15.8 \\
$e_{15}\left(\mathrm{C} \mathrm{m}^{-2}\right)$ & 12.3 \\
$\epsilon_{11}^{S} / \epsilon_{0}$ & 1650 \\
$\epsilon_{33}^{S} / \epsilon_{0}$ & 1700 \\
$\rho\left(\mathrm{kg} \mathrm{m}^{-3}\right)$ & 5000 \\
Steel & \\
$c_{11}\left(10^{11} \mathrm{~N} \mathrm{~m}^{-2}\right)$ & 3.11 \\
$c_{12}\left(10^{11} \mathrm{~N} \mathrm{~m}^{-2}\right)$ & 1.53 \\
$\rho\left(\mathrm{kg} \mathrm{m}^{-3}\right)$ & 7800 \\
\hline
\end{tabular}

domain consists of piezoceramic (no steel at all) and has a square shape. For both configurations, the piezoceramic is polarized in the 3 direction, as shown in figure 5. Table 1 describes the properties of the piezoceramic (PZT5A) and steel used in the simulations. For resonator applications the piezoelectric material quartz would be preferred to PZT5A due to its highly stable properties. However, since we are only interested in showing the design method, PZT5A was considered for the sake of simplicity.
As an initial guess, the design variable $x_{n}$ is set to be equal to 0.5 in both models. When the optimization process is complete, the result is a density distribution over the mesh with some 'gray scale' (densities between zero and one). Even though the cost constraint implemented reduces the amount of gray scale, it is difficult to eliminate it entirely since the optimum solution requires some gray scale [17]. However, we need to interpret the results as a distribution of two phases by eliminating the intermediate densities which are difficult to implement in practice. This elimination can be implemented by using techniques such as image processing [11]. Independent of the interpretation technique used to obtain a clean structure, there will always be a change in the frequencies and objective function (EMCC for example) since the gray scale is no longer present. Therefore, another optimization process, such as shape optimization [18] which produces small changes in the topology, would be necessary to recover the objective function improvement and also to provide a final design that can be manufactured.

The interpretation problem is less critical when we are only maximizing (or minimizing) an objective function, since at most the objective function will decrease (or increase) due to the elimination of 'gray scale'. However, it becomes critical, for example, in the case where we specify the desired frequencies for the transducer design since small changes in the structure may cause the final resonance frequencies to deviate from the desired values. In this work, a simple threshold was applied to the density $x_{n}$ of the optimized topology to obtain the final topologies shown in the pictures.

The first design considered is the maximization of the EMCC for a specific mode or more than one mode. Figure 6 shows a transducer topology that maximizes the EMCC for the second vibration mode considering only piezoceramic material in the design domain. Figure 8 shows the design of the steel coupling structure that maximizes the EMCC (or $k$ ) for the first mode using model PZT5A/steel. The graphs showing the iteration history for the EMCCs $(k)$, cost constraint $(W)$ and objective function $\left(F_{1}\right)$ for the previous designs are shown in figures 6 and 9 , respectively. Six modes $(m=6)$ were used in equation (4) for all results shown. The weights $\left(w_{i}\right)$ in equation (4) were chosen to be 1000 for the $k$ coefficients we want to maximize and one for the others. 

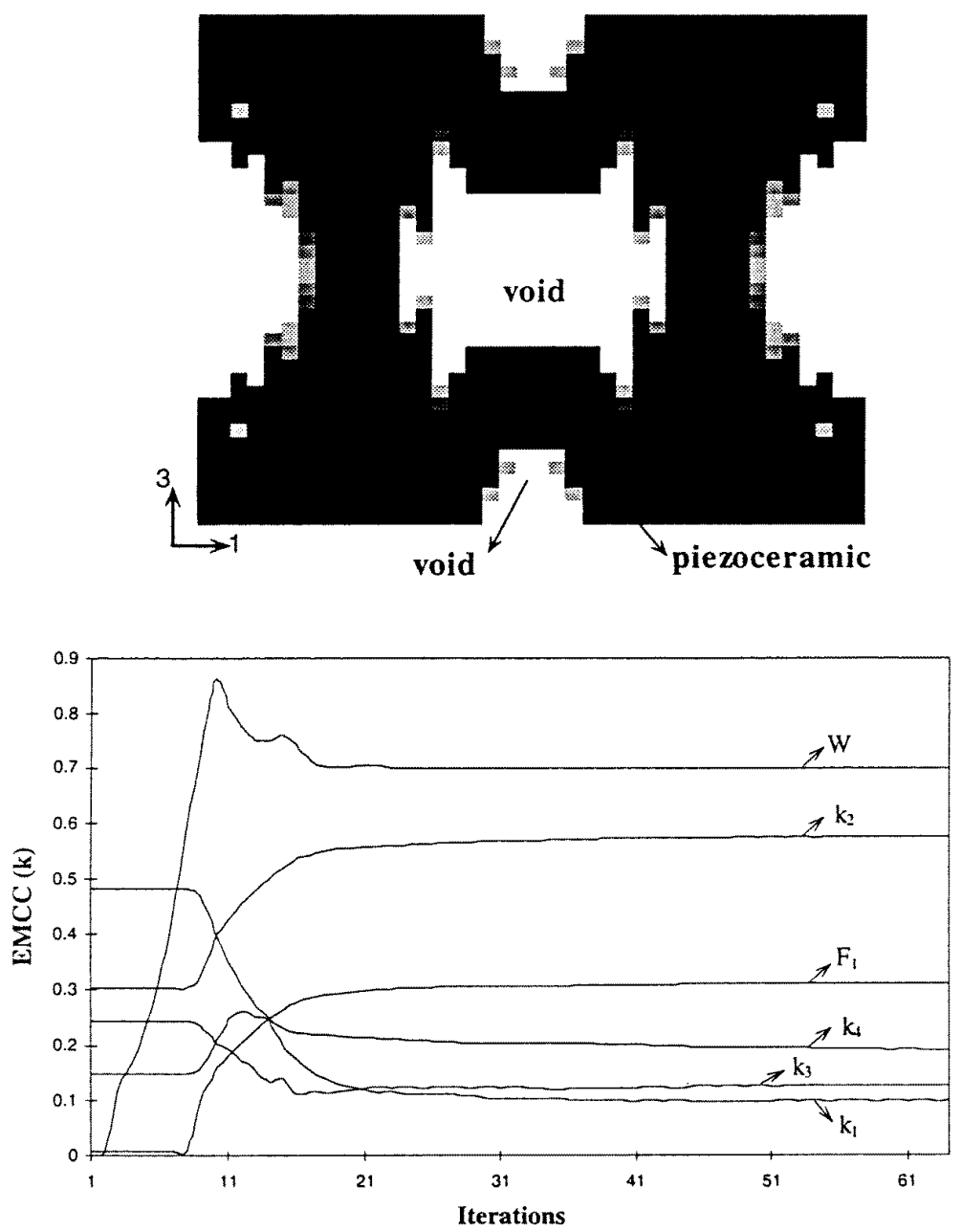

Figure 6. Transducer topology that maximizes $k$ for the second mode. Only piezoceramic is considered in the domain. Iteration history for the first four $k$, objective function $\left(F_{1}\right)$ and constraint $(W)$ is also presented.

Table 2. Electromechanical coupling coefficient $(k)$ for the first four modes of the transducers described in the figures. An asterisk $(*)$ indicates maximized $k$. Constraint $W$ is also given for each design.

\begin{tabular}{lllll}
\hline Design & Figure 5 & Figure 6 & Figure 8 & Figure 10 \\
\hline$k_{1}$ & 0.425 & 0.128 & $0.501^{*}$ & $0.362^{*}$ \\
$k_{2}$ & 0.082 & $0.580^{*}$ & 0.261 & $0.354^{*}$ \\
$k_{3}$ & 0.120 & 0.123 & 0.135 & $0.346^{*}$ \\
$k_{4}$ & 0.194 & 0.181 & 0.179 & 0.271 \\
$W>$ & - & 0.7 & 0.7 & 0.75 \\
\hline
\end{tabular}

The corresponding 'piston' vibration modes, ideal for generating acoustic waves, are presented for these topologies in figures 7 and 8, respectively, considering only one quarter of the domain (due to the symmetry). These modes are important if the transducer will be applied for generation and reception of acoustic waves. Figure 10 presents the resultant transducer topology that simultaneously maximizes the EMCC for the first three vibration modes. The iteration history is also presented. This gives a plurimodal behavior in the transducer frequency response.

Table 2 describes the values for the first four electromechanical coupling coefficients $(k)$ for the transducers pre- sented in the figures and also for the initial domain described in figure 5 (considering $x_{n}=1$ ). By comparing the value of the EMCC achieved for figure 6 with the values described in the dispersion curve in figure 4 , we can see that the improvement obtained is larger than the one obtained if only the dimension rate is changed for the simple rectangular shape. Also, comparing the EMCC for figure 8 with the transducer without the coupling structure (see dispersion curve for $W / T=2.33$ ), we can verify the improvement in the EMCC due to the coupling structure with complex topology added to the PZT transducer. Notice also that almost all of the 'piston' modes described above have high values of EMCC which indicates a high coupling of these modes with the excitation.

Sometimes, the goal could be to design a transducer with the same value of EMCC as given in dispersion curve 4 for a specific aspect ratio $(W / T)$, but considering a different aspect ratio of the design domain. An application of this would be the design of piezoelectric elements for 1-3 piezocomposite transducers. In these transducers, considering parallelepipedic shape elements, a good performance usually can be achieved only for a ratio width/thickness around $0.6[4,6]$. This makes it difficult to build these piezocomposites for low volume fractions without increasing the lateral periodicity of the piezocomposite in 


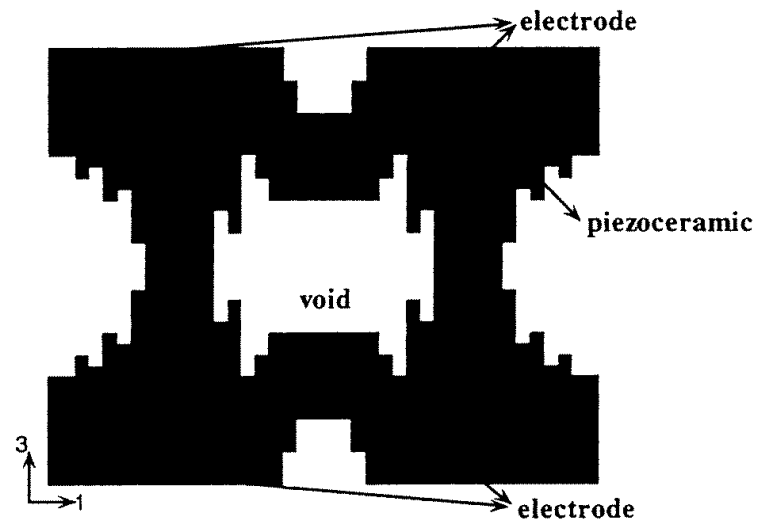

a) Transducer Topology

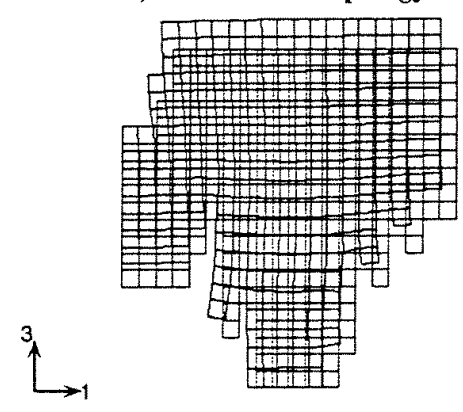

b) Second Mode

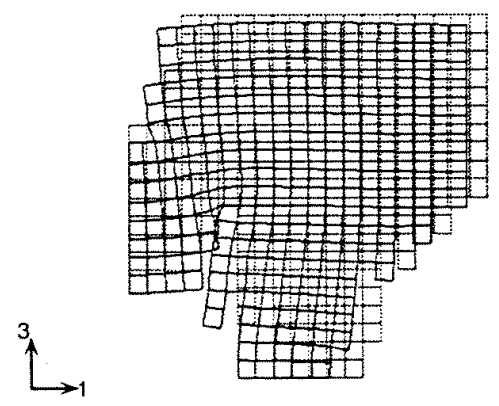

c) Third Mode

Figure 7. Thresholded image of the topology in figure 6 . The corresponding second and third resonance modes are also presented using a $20 \times 20$ mesh for one quarter of the domain. Dashed lines indicate undeformed structure.

relation to the thickness (equal to operational wavelength). If this happens, scattering will occur inside the piezocomposite making it difficult to model its behavior [19]. A new design for the piezoelectric element within a domain aspect ratio constraint may be sought to avoid this limitation.

The next step consists of designing a transducer topology with specified frequencies. This is an important goal in the transducer design, since it allows us, for example, to place some undesired modes (such as spurious modes) far from the transducer operational mode, as described in [2]. The presence of these modes close to the operational frequency could cause some undesired change in the resonator operational frequency, for example.

Figures 11, 13 and 15 show the topologies obtained for the specified resonance frequencies described in table 3, which also shows the initial frequencies and the achieved resonance frequencies. Figures 11 and 13 present transducer

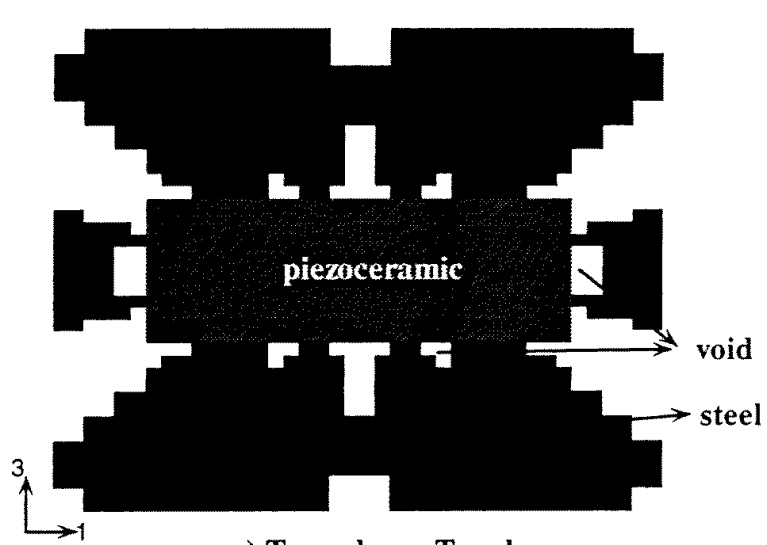

a) Transducer Topology

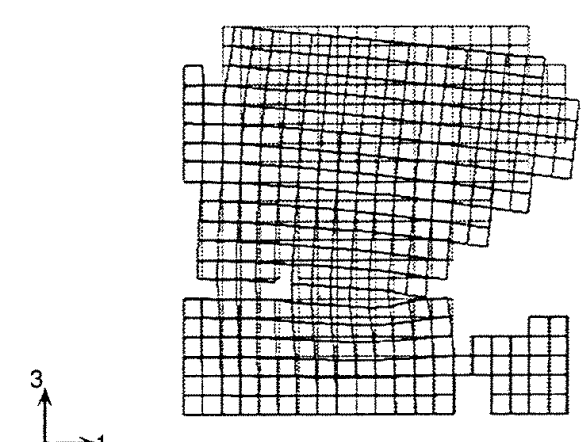

b) First Mode

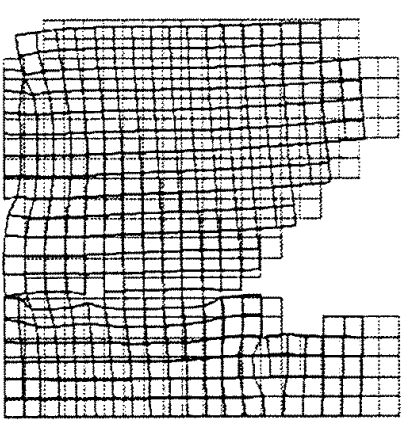

c) Third Mode

Figure 8. Transducer topology that maximizes $k$ for the first mode considering PZT5A/steel configuration. Piezoceramic domain remains unchanged. The corresponding first and third resonance modes are also presented using a $20 \times 20$ mesh for one quarter of the domain. Dashed lines indicate undeformed structure.

Table 3. Initial, specified and achieved resonance frequencies for the transducer designs shown in the figures.

\begin{tabular}{lllcc}
\hline Design & & Figure 11 & Figure 13 & Figure 15 \\
\hline \multirow{2}{*}{ Initial } & $\omega_{r 1}$ & 4.6 & 4.6 & 5.7 \\
$10^{3}\left(\right.$ cycles s$\left.^{-1}\right)$ & $\omega_{r 2}$ & 5.9 & 5.9 & 8.3 \\
& $\omega_{r 3}$ & 8.4 & 8.4 & 10.5 \\
Specified & $\omega_{r 1}$ & 3.0 & 4.58 & 4.0 \\
$10^{3}\left(\right.$ cycles s$\left.^{-1}\right)$ & $\omega_{r 2}$ & 7.0 & 12.0 & 7.0 \\
& $\omega_{r 3}$ & 9.0 & 16.0 & 9.0 \\
Achieved & $\omega_{r 1}$ & 3.03 & 4.97 & 4.07 \\
$10^{3}\left(\right.$ cycles s $\left.^{-1}\right)$ & $\omega_{r 2}$ & 6.99 & 10.5 & 7.03 \\
\hline
\end{tabular}

designs considering only piezoceramic in the domain, while 


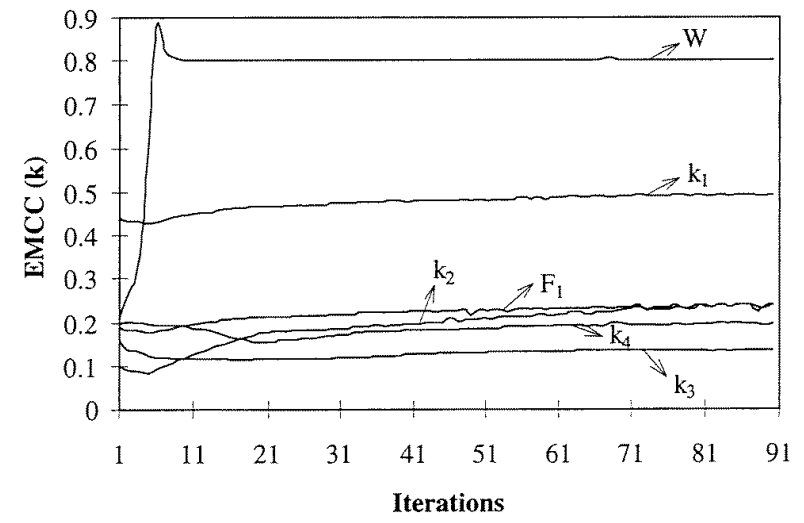

Figure 9. Iteration history for first four $k$, objective function $\left(F_{1}\right)$ and constraint $(W)$ for figure 8 .
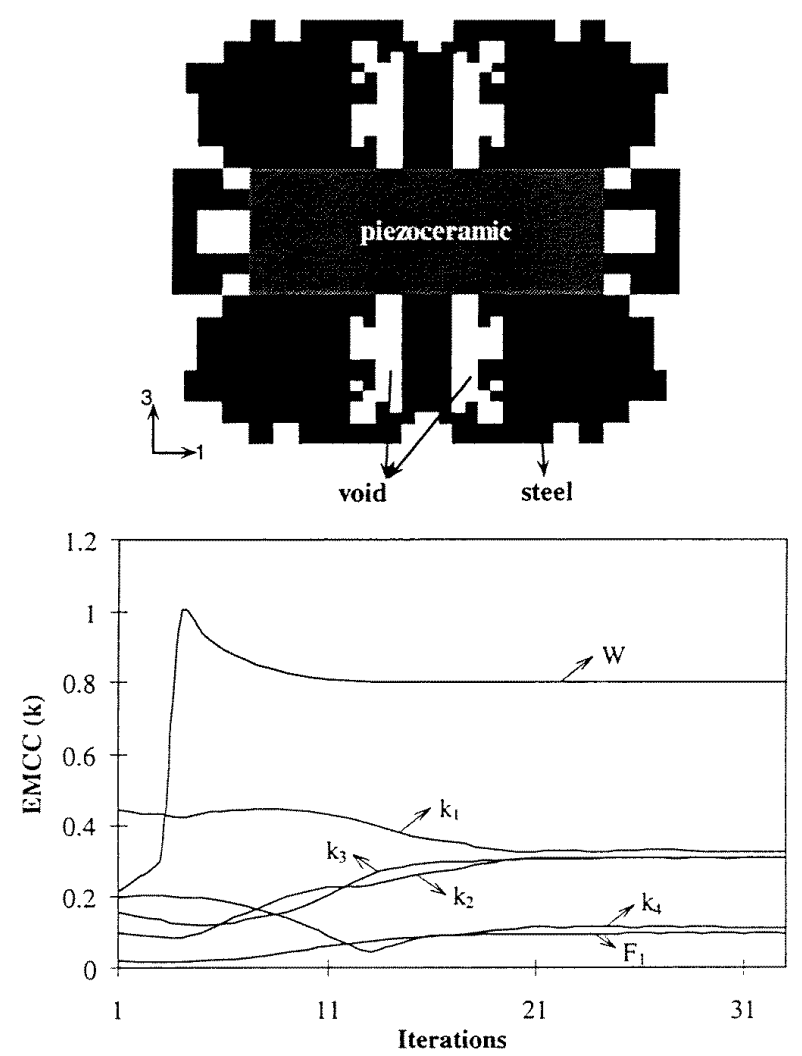

Figure 10. Transducer topology that maximizes $k$ for the first three modes considering the PZT5A/steel configuration.

Piezoceramic domain remains unchanged. Iteration history for $k$, objective function $\left(F_{1}\right)$ and constraint $(W)$ is also presented.

figure 15 considers model PZT5A/steel (piezoceramic is kept unchanged). The graphs showing the iteration history for the resonance and antiresonance frequencies for the designs in figures 11 and 13 are shown in figures 12 and 14, respectively. A graph showing the iteration history for cost constraint $(W)$, and objective function $\left(F_{2}\right)$ for figure 11 is shown in figure 12 . Three modes $(m=3)$ were used in equation (5) for all results shown. The corresponding 'piston' vibration modes, ideal for generating acoustic waves, are presented for these topologies in figures 11, 13 and 15, respectively, considering only one quarter of the domain (due to the symmetry). As discussed,

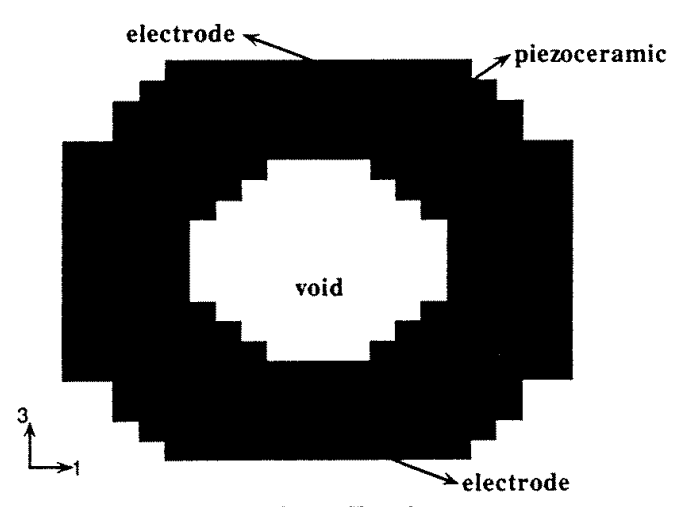

a) Transducer Topology

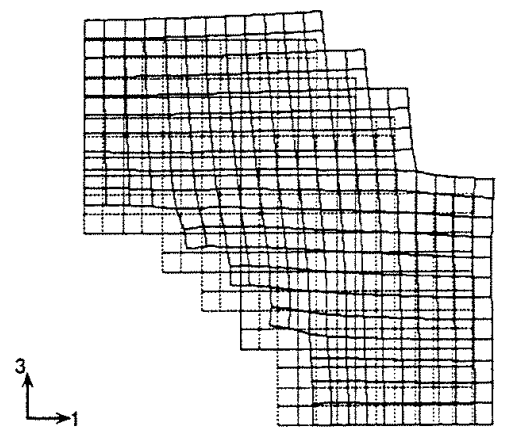

b) Second Mode

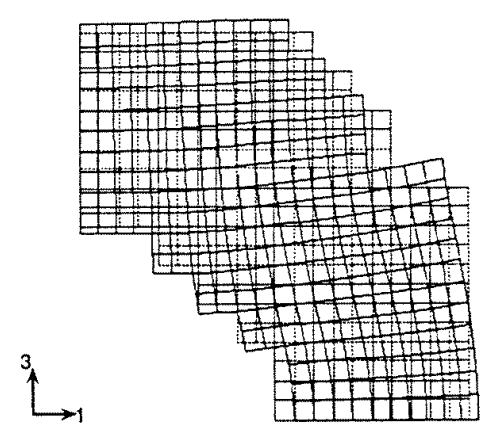

c) Third Mode

Figure 11. Transducer topology obtained for specified resonance frequencies described in table 3 . Only piezoceramic is considered in the domain. The corresponding second and third resonance modes are also presented using a $20 \times 20$ mesh for one quarter of the domain. Dashed lines indicate undeformed structure.

Table 4. Antiresonance frequencies and electromechanical coupling coefficient $(k)$ for the first three modes of the transducers described in the figures.

\begin{tabular}{lllcl}
\hline Design & & Figure 11 & Figure 13 & Figure 15 \\
\hline \multirow{3}{*}{ Antiresonance } & $\omega_{s 1}$ & 3.17 & 5.38 & 4.35 \\
$10^{3}\left(\right.$ cycles s $\left.^{-1}\right)$ & $\omega_{a 3}$ & 7.16 & 10.81 & 7.32 \\
& $k_{1}$ & 0.308 & 11.47 & 9.35 \\
EMCC $(k)$ & $k_{2}$ & 0.222 & 0.415 & 0.377 \\
& $k_{3}$ & 0.365 & 0.245 & 0.290 \\
Constraint & $W>$ & 0.7 & 0.7 & 0.236 \\
\hline
\end{tabular}

these are ideal for generating acoustic waves if the transducer is applied for acoustic wave generation.

Table 4 presents the first three antiresonance frequencies and EMCCs for these transducers, as well as the constraint 

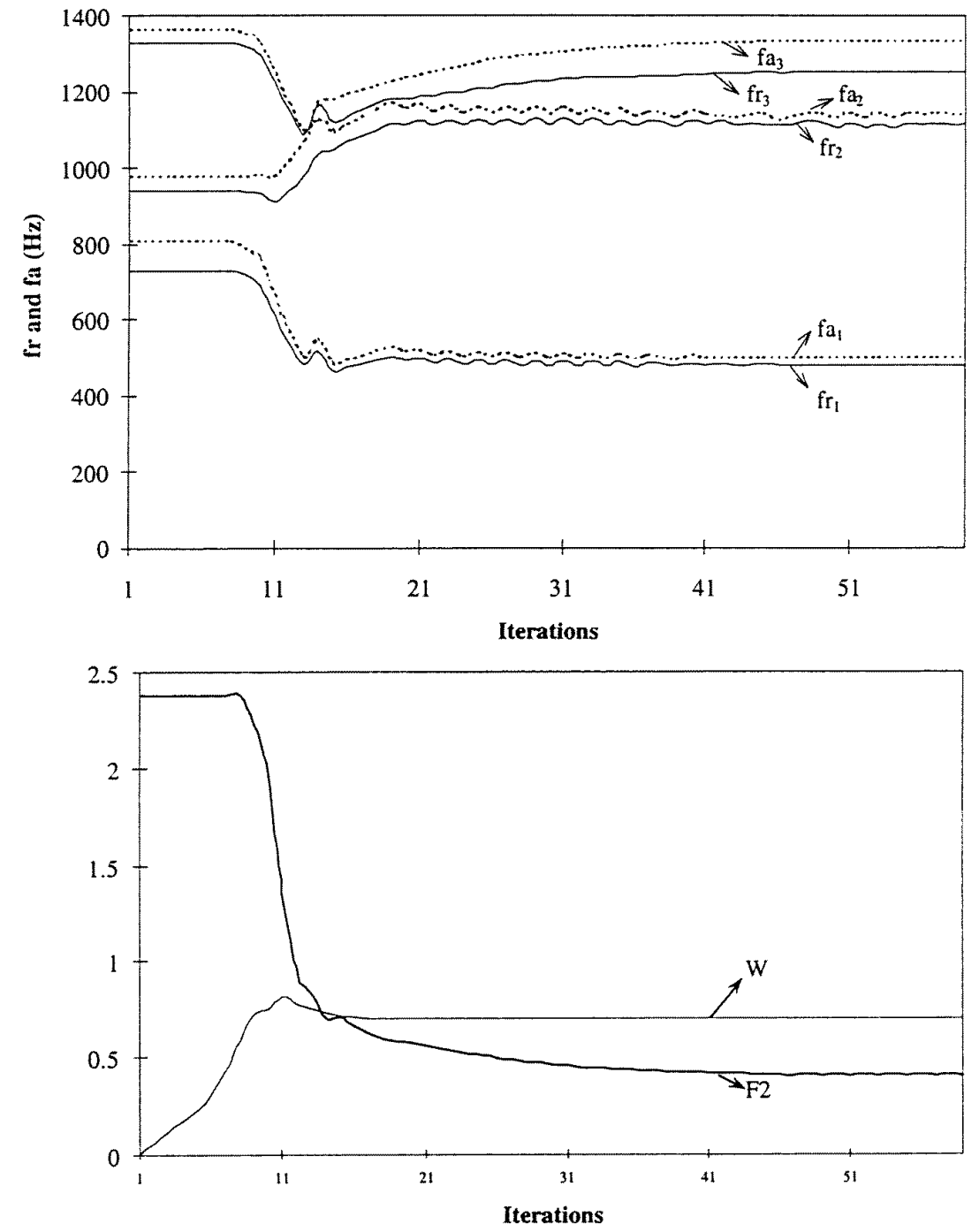

Figure 12. Iteration history for resonance $\left(f_{r}\right)$ and antiresonance $\left(f_{a}\right)$ frequencies, objective function $\left(F_{2}\right)$ and constraint $(W)$ for figure 11 .

$W$ specified in the optimization. We can see that the 'piston' modes described above have high values of EMCC which indicates a high coupling of these modes with the excitation. Notice that the frequency units are $\mathrm{Hz}$ in the graphs and cycles $\mathrm{s}^{-1}$ (equal to $2 \pi \mathrm{Hz}$ ) in the tables. The gap among the desired frequencies and achieved frequencies in figures 11 and 13 is probably due to the small relaxation given by the material model used, therefore not all combinations of specified frequencies can be achieved.

As discussed above, the final interpretation of this design is critical since small changes in the topology may cause the final frequencies to deviate from the desired ones; however, this kind of objective function plays an important role in the design of a narrowband (or a broadband) transducer (the next design considered) since usually we are more interested in making the resonance frequencies far from (or close to) each other (as explained above), rather than equal to specific values.

The final design considered is the design of a narrowband and a broadband transducer. It consists of combining the previous objective functions: maximization of the EMCC ( $k$ ) for a specific mode or modes and design of the transducer with specified frequencies. Of course, in this multiobjective function the result will be a compromise between these two goals, which means that the desired frequencies will not be achieved exactly nor will the EMCC be maximized as much as in the first case, depending on the chosen weight $\beta$.

Figure 16 shows a transducer with the first resonance far from the others and with the largest EMCC, as described in the EMCC response spectrum in figure 17. This provides a narrowband behavior in the transducer response. The corresponding two 'piston' mode shapes, considering only one quarter of the domain (due to the symmetry), are presented in figure 16. Figure 18 shows the topology of a transducer with a broadband frequency response. The three resonance frequencies are close to each other, each of them with large $k$. This example illustrates the application of the third objective function in the design of a multimode broadband Tonpilz transducer [12] as discussed before.

The graphs showing the iteration history for the resonance and antiresonance frequencies, EMCCs, cost constraint $(W)$ and objective function $\left(F_{3}\right)$ for the design in figure 18 are shown in figure 19. The corresponding 'piston' 


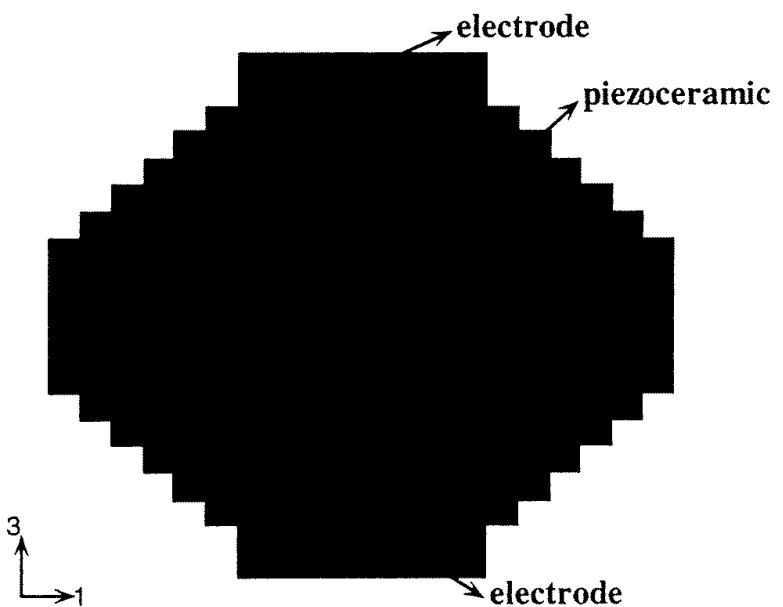

a) Transducer Topology

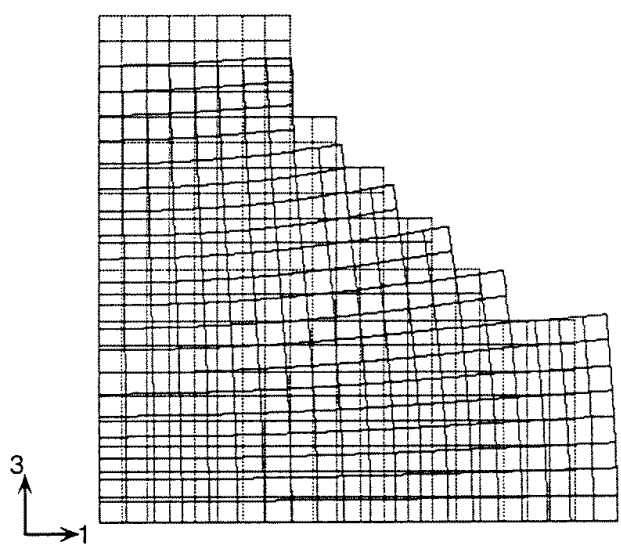

b) First Mode

Figure 13. Transducer topology obtained for specified resonance frequencies described in table 3 . Only piezoceramic is considered in the domain. The corresponding first resonance mode is also presented using $20 \times 20$ mesh for one quarter of the domain. Dashed lines indicate undeformed structure.

Table 5. Initial, specified and achieved resonance frequencies for the transducer designs shown in the figures.

\begin{tabular}{lccc}
\hline Design & & Figure 16 & Figure 18 \\
\hline \multirow{3}{*}{ Initial } & $\omega_{r 1}$ & 4.6 & 5.7 \\
$10^{3}\left(\right.$ cycles s $\left.^{-1}\right)$ & $\omega_{r 2}$ & 5.9 & 8.3 \\
& $\omega_{r 3}$ & 8.4 & 10.5 \\
Specified & $\omega_{r 1}$ & 3.0 & 3.0 \\
$10^{3}\left(\right.$ cycles s $\left.^{-1}\right)$ & $\omega_{r 2}$ & 7.6 & 4.6 \\
& $\omega_{r 3}$ & 12.3 & 6.0 \\
Achieved & $\omega_{r 1}$ & 3.09 & 3.07 \\
$10^{3}\left(\right.$ cycles s$\left.^{-1}\right)$ & $\omega_{r 2}$ & 6.62 & 4.40 \\
\hline
\end{tabular}

mode shapes, considering only one quarter of the domain (due to the symmetry), are presented in figure 18.

Table 5 describes the first three initial, specified and achieved resonance frequencies and specified constraint for the topologies presented. Table 6 presents the corresponding first three antiresonance frequencies and $k$ for these transducers. The weight $(\beta)$ used in the multiobjective function $F_{3}(6)$ is 0.3 and 0.8 for the designs in figures 16

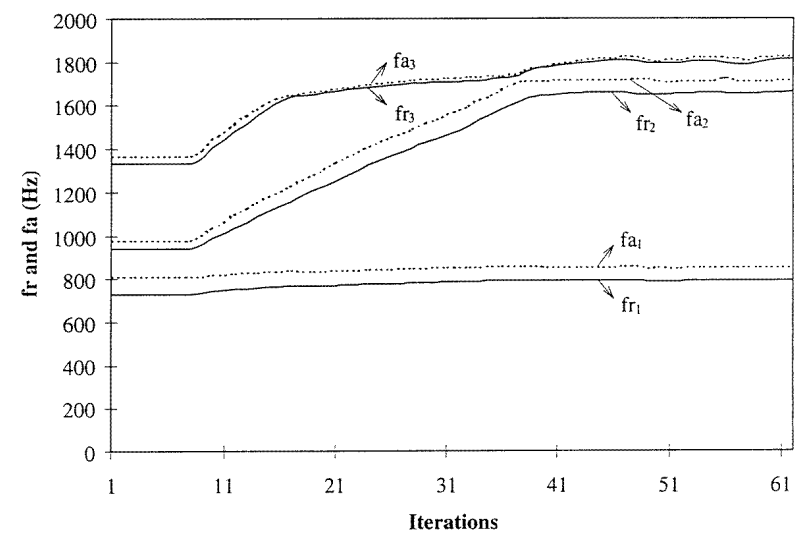

Figure 14. Iteration history for resonance $\left(f_{r}\right)$ and antiresonance $\left(f_{a}\right)$ frequencies for figure 13 .

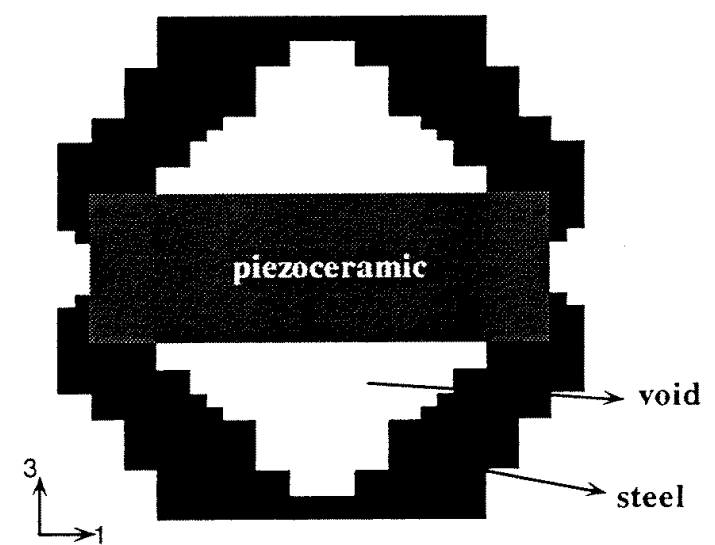

a) Transducer Topology

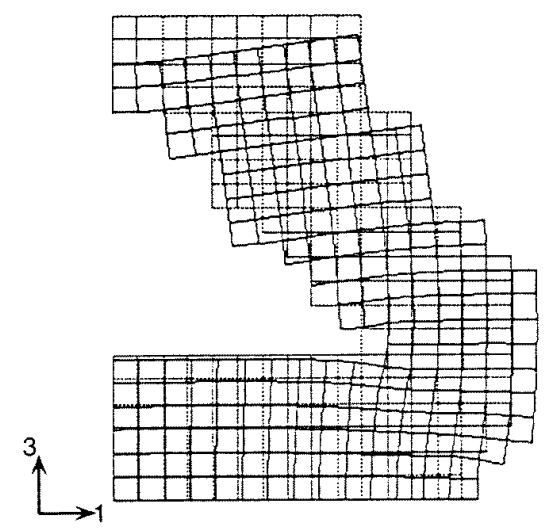

b) First Mode

Figure 15. Transducer topology obtained for specified resonance frequencies described in table 3 considering the PZT5A/steel configuration. The corresponding first resonance mode is also presented using a $20 \times 20$ mesh for one quarter of the domain. Dashed lines indicate undeformed structure.

and 18 , respectively. The weights $\left(w_{i}\right)$ of $F_{2}$ in equation (6) were chosen to be 10000 for the $k$ coefficients we want to maximize and one for the others. Five modes were used in equation $(6)(m=5)$ for figure 16 , and three modes $(m=3)$ for figure 18. Notice that the first 'piston' mode in figure 16 and the second one in figure 18 have the largest EMCC values showing a high coupling with the excitation. 


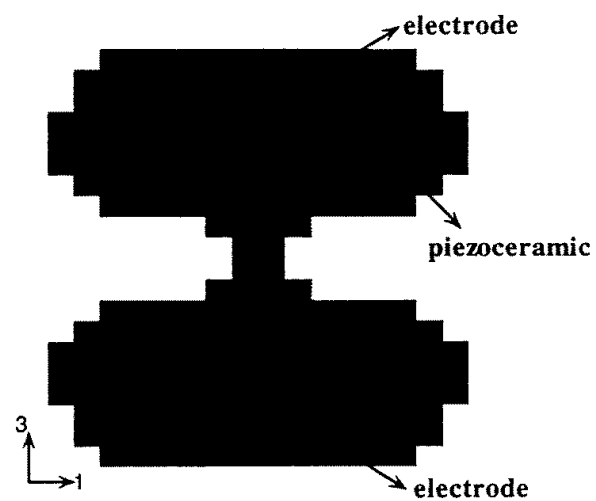

a) Transducer Topology

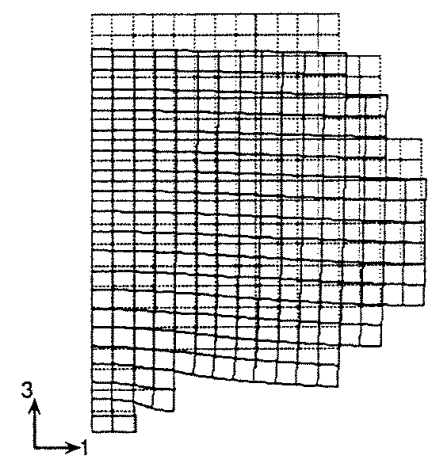

b) First Mode

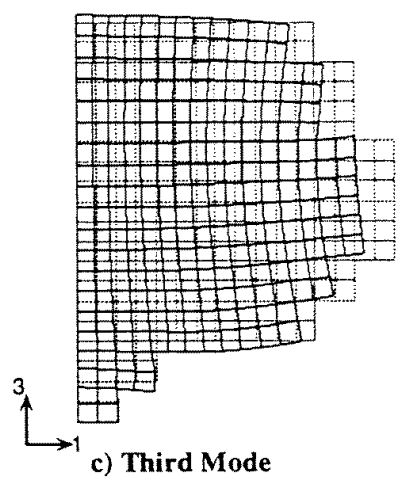

Figure 16. Transducer topology obtained using the third objective function. Only piezoceramic is considered in the domain. The corresponding first and third resonance modes are also presented using a $20 \times 20$ mesh for one quarter of the domain. Dashed lines indicate undeformed structure.

Table 6. Electromechanical coupling coefficient $(k)$ for the first three modes of the transducers described in the figures. An asterisk $(*)$ indicates maximized $k$.

\begin{tabular}{llll}
\hline Design & & Figure 16 & Figure 18 \\
\hline \multirow{3}{*}{ Antiresonance } & $\omega_{a 1}$ & 3.49 & 3.18 \\
$10^{3}\left(\right.$ cycles s$\left.^{-1}\right)$ & $\omega_{a 3}$ & 7.64 & 4.82 \\
& $k_{1}$ & $0.53^{*}$ & 5.98 \\
EMCC $(k)$ & $k_{2}$ & 0.08 & $0.47^{*}$ \\
& $k_{3}$ & 0.1 & $0.25^{*}$ \\
Constraint & $W>$ & 0.6 & 0.7 \\
\hline
\end{tabular}

\section{Conclusions}

A method for designing piezoelectric transducers with high performance characteristics has been proposed. It is based 362

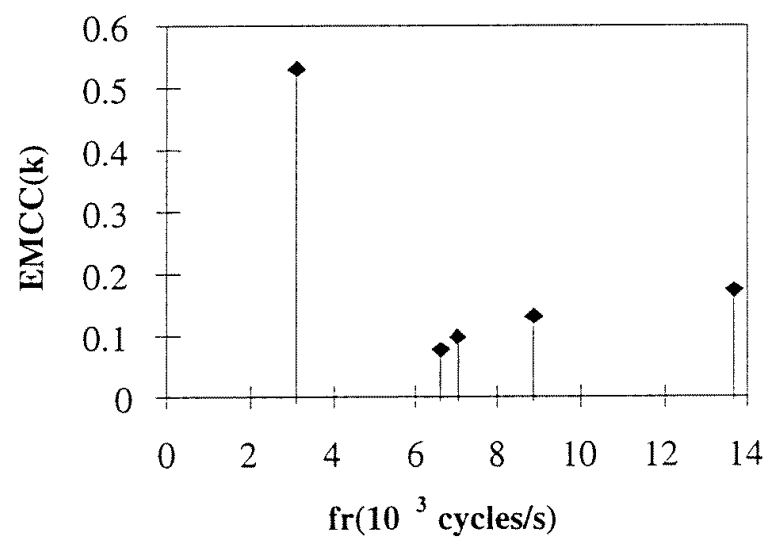

Figure 17. EMCC response spectrum for the transducer in figure 16. $f_{r}$ is the resonance frequency.

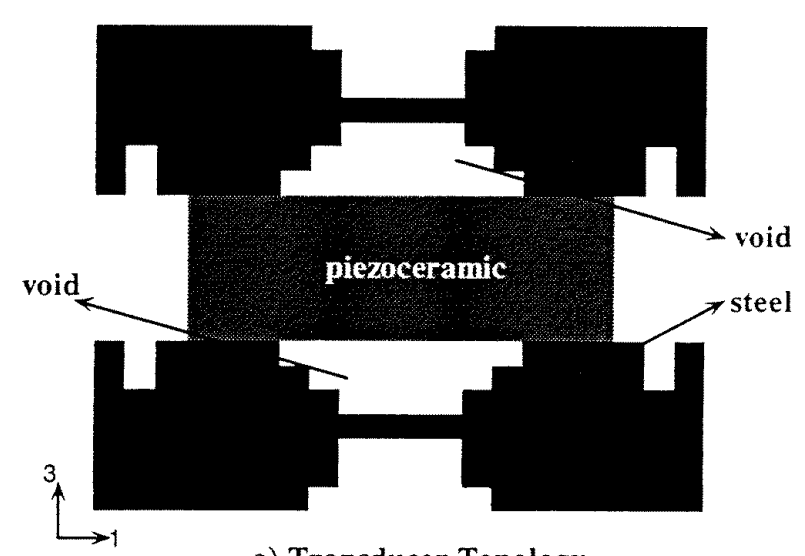

a) Transducer Topology

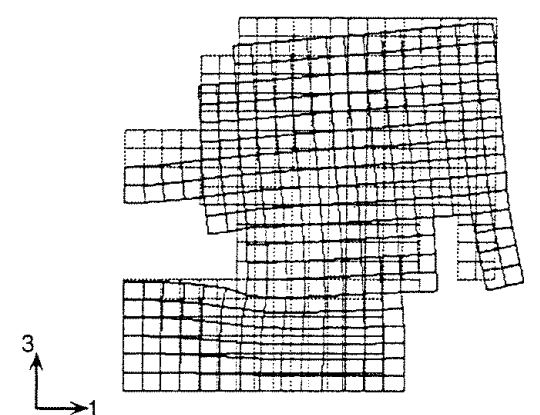

b) Second Mode

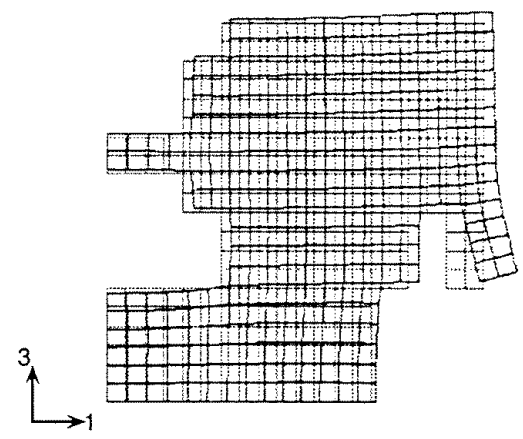

c) Third Mode

Figure 18. Transducer topology obtained using the third objective function considering the PZT5A/steel configuration. The corresponding second and third resonance modes are also presented using a $20 \times 20$ mesh for one quarter of the domain. Dashed lines indicate undeformed structure. 

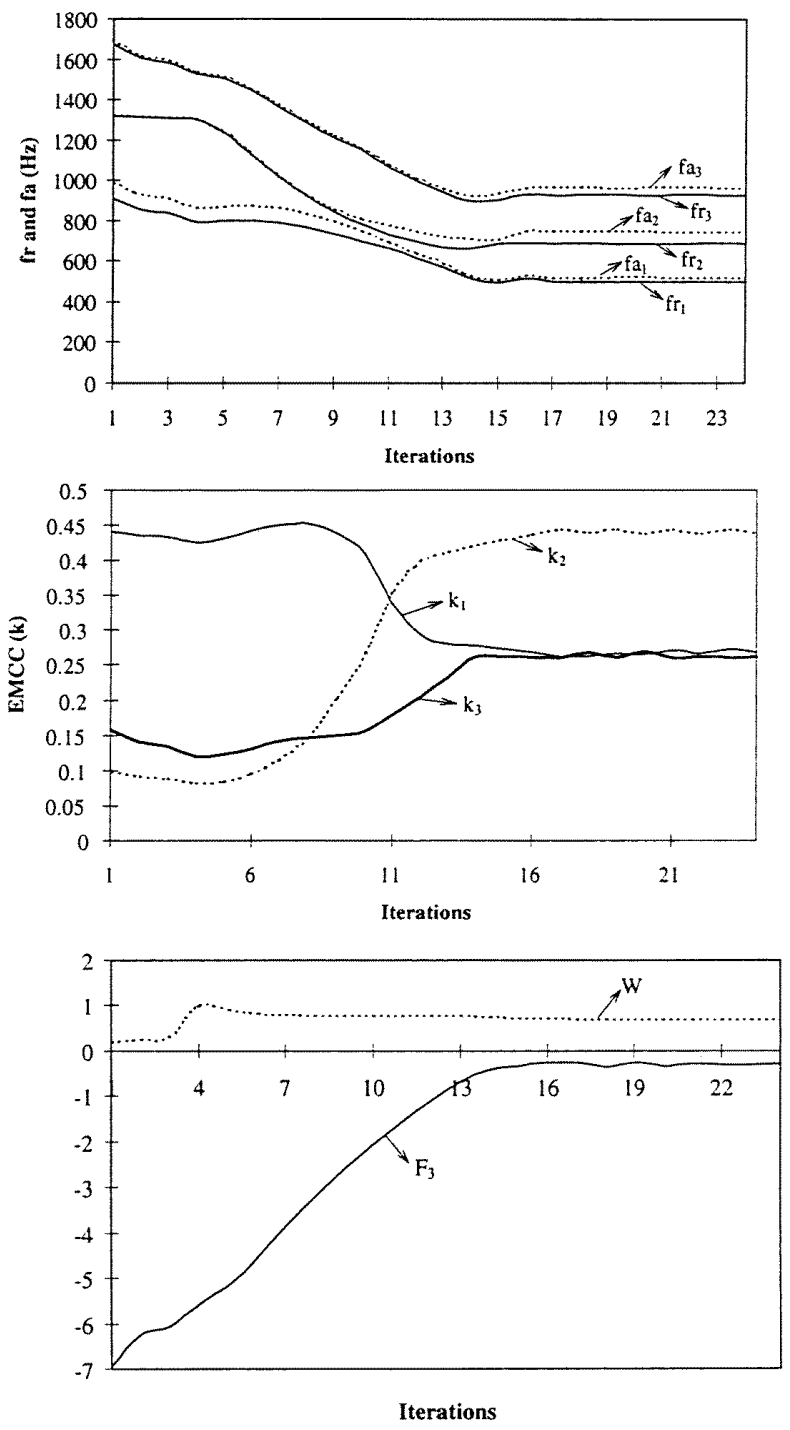

Figure 19. Iteration history for resonance $\left(f_{r}\right)$ and antiresonance $\left(f_{a}\right)$ frequencies, EMCCs $(k)$, objective function $\left(F_{3}\right)$ and constraint $(W)$ for figure 18 .

upon topology optimization techniques and the finite element method (FEM). Three kinds of objective function were defined: maximization of the response of a specific mode of the transducer operation (by maximizing its EMCC), design of a transducer with specified resonance frequencies and design of the transducer frequency response spectrum (narrowband or broadband transducer). This method can be applied to design transducers for acoustic wave generation and resonator applications.

The proposed design method allows us to obtain transducers with specified frequencies and better performance characteristics than the ones achieved with simple geometric shapes. Improvement of the electromechanical coupling factor (EMCC) was obtained in relation to simple designs of piezoelectric transducers (such as a rectangular shape); however, complex piezoceramic topologies are obtained as a result. The manufacture of these complex ceramic shapes can be achieved by using rapid prototyping techniques that have been developed by [20]. The model PZT5A/steel is an

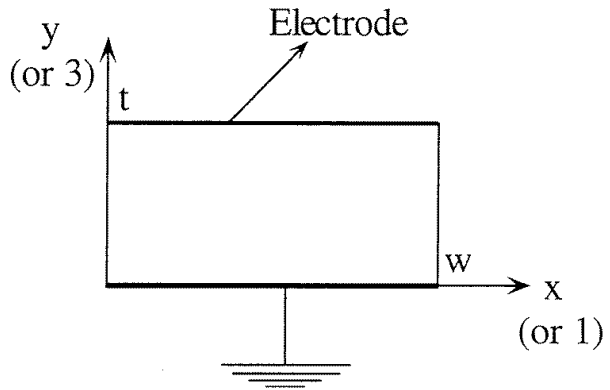

Figure A1. Design domain considering one quarter symmetry.

alternative approach that allows us to change the transducer characteristics by adding a coupling structure with complex topology to a simple shape PZT transducer, which is easier to manufacture. This model can be used for the design of a Tonpilz transducer [12].

In future work, we intend to extend this method to 3D transducer design. A shape optimization applied to the piezoelectric medium [18] must be implemented in order to be able to obtain a clear final design. The material model can be changed for a more complex one (microstructure defined in [10]) which enlarges the solution space of the problem (increases the problem 'relaxation'). Furthermore, other kinds of objective function, or modifications of the current ones, can be implemented depending on the specific goal of the piezoelectric transducer application and other initial design domains can be considered.

\section{Acknowledgments}

The first author thanks CNPq-Conselho Nacional de Desenvolvimento Científico e Tecnológico (Brazilian National Council for Scientific and Technologic Development) - and University of São Paulo (Brazil) for supporting him in his graduate studies. The authors are thankful for the financial support received through a Maxwell project (ONR 0001494-1-022 and US Army TACOM DAAE 07-93-C-R125).

\section{Appendix A. Symmetry conditions}

In this appendix, we present the symmetry conditions imposed in the FEM model that allow us to solve for the resonance and antiresonance frequencies by considering only one quarter of the transducer domain. The domain is shown in figure $\mathrm{A} 1$ and the specified boundary conditions are described in table A1 in terms of displacements and electrical potential degrees of freedom.

Table A1. Boundary conditions for the FEM model considering one quarter symmetry. $u_{i}$ is the displacement in the $i$ direction and $\phi$ is the electrical potential.

\begin{tabular}{lll}
\hline Side & Resonance & Antiresonance \\
\hline$\gamma=0$ & $u_{\gamma}=0 ; \phi=0$ & $u_{\gamma}=0 ; \phi=0$ \\
$y=t$ & $\phi=0$ & equipotential \\
$x=0$ & $u_{x}=0$ & $u_{x}=0$ \\
\hline
\end{tabular}




\section{Appendix B. Sensitivity analysis}

\section{Appendix B.1. Sensitivity of the resonance and antiresonance eigenvalues}

The resonance and antiresonance eigenvalues are obtained by solving the eigenvalue problems resulting from applying boundary conditions to equation (1):

$$
(\overline{\mathbf{K}}-\lambda \overline{\mathbf{M}}) \boldsymbol{W}=\mathbf{0} ; \text { and } \boldsymbol{W}=\left\{\begin{array}{c}
\boldsymbol{U} \\
\boldsymbol{\Phi}
\end{array}\right\} \quad \lambda=\omega^{2}
$$

where $\overline{\mathbf{K}}$ and $\overline{\mathbf{M}}$ are the 'total' stiffness and mass matrices involving electrical and mechanical degrees of freedom that take into account the boundary conditions of the resonance or antiresonance problem (see appendix A).

The sensitivity of the resonance and antiresonance eigenvalues is calculated by deriving equation (10) in relation to the design variables $x_{n}$ following a procedure similar to that described in [21]. For the resonance eigenvalue $\lambda_{r}$, we obtain

$$
\frac{\partial \lambda_{r}}{\partial x_{n}}=\frac{\boldsymbol{W}_{r}^{T}\left(\frac{\partial \overline{\mathbf{K}}_{r}}{\partial x_{n}}-\lambda_{r} \frac{\partial \overline{\mathbf{M}}_{r}}{\partial x_{n}}\right) \boldsymbol{W}_{r}}{\boldsymbol{W}_{r}^{T} \overline{\mathbf{M}}_{r} \boldsymbol{W}_{r}} \quad \lambda_{r}=\omega_{r}^{2}
$$

For the antiresonance eigenvalue $\lambda_{a}$, we obtain:

$$
\frac{\partial \lambda_{a}}{\partial x_{n}}=\frac{\boldsymbol{W}_{a}^{T}\left(\frac{\partial \overline{\mathbf{K}}_{a}}{\partial x_{n}}-\lambda_{a} \frac{\partial \overline{\mathbf{M}}_{a}}{\partial x_{n}}\right) \boldsymbol{W}_{a}}{\boldsymbol{W}_{a}^{T} \overline{\mathbf{M}}_{a} \boldsymbol{W}_{a}} \quad \lambda_{a}=\omega_{a}^{2}
$$

where $\overline{\mathbf{K}}_{r}, \overline{\mathbf{M}}_{r}, \overline{\mathbf{K}}_{a}$ and $\overline{\mathbf{M}}_{a}$ are the total stiffness and mass matrices reduced for the boundary conditions of the resonance and antiresonance problems, respectively, and $\boldsymbol{W}_{r}$ and $\boldsymbol{W}_{a}$ are the resonance and antiresonance modes obtained from the solution of the eigenvalue problem (10) for different boundary conditions.

Due to the material assumption described in section 3.2 the matrices $\partial \overline{\mathbf{K}}_{r} / \partial x_{n}, \partial \overline{\mathbf{M}}_{r} / \partial x_{n}, \partial \overline{\mathbf{K}}_{a} / \partial x_{n}$ and $\partial \overline{\mathbf{M}}_{a} / \partial x_{n}$ are proportional to the individual element matrices $\left[\overline{\mathbf{K}}_{r}\right]_{n},\left[\overline{\mathbf{M}}_{r}\right]_{n}$, $\left[\overline{\mathbf{K}}_{a}\right]_{n}$ and $\left[\overline{\mathbf{M}}_{a}\right]_{n}$, and are given by the expressions:

$$
\begin{array}{rlrl}
\frac{\partial \overline{\mathbf{K}}_{r}}{\partial x_{n}} & =\frac{\left[\overline{\mathbf{K}}_{r}\right]_{n}}{x_{n}} & \frac{\partial \overline{\mathbf{M}}_{r}}{\partial x_{n}}=\frac{\left[\overline{\mathbf{M}}_{r}\right]_{n}}{x_{n}} \\
\frac{\partial \overline{\mathbf{K}}_{a}}{\partial x_{n}}=\frac{\left[\overline{\mathbf{K}}_{a}\right]_{n}}{x_{n}} & \frac{\partial \overline{\mathbf{M}}_{a}}{\partial x_{n}}=\frac{\left[\overline{\mathbf{M}}_{a}\right]_{n}}{x_{n}} .
\end{array}
$$

\section{Appendix B.2. Sensitivity of objective functions}

The sensitivity of objective functions can be obtained by deriving equations (4), (5) and (6) in relation to design variables $x_{n}$ and expressing the result as a function of the sensitivity of the resonance and antiresonance eigenvalues. The derivation of equations (4), (5) and (6) is straightforward; therefore, it will not be presented here.

\section{References}

[1] Kunkel H A, Locke S and Pikeroen B 1990 Finite element analysis of vibrational modes in piezoelectric ceramic disks IEEE Trans. Ultrason. Ferroelectr. Freq. Control 37 316-28
[2] Simson É A and Taranukha A 1993 Optimization of the shape of a quartz resonator Acoust. Phys. 39 472-6

[3] Challande P 1990 Optimizing ultrasonic transducers based on piezoelectric composites using a finite-element method IEEE Trans. Ultrason. Ferroelectr. Freq. Control 37 $135-40$

[4] Sato J, Kawabuchi M and Fukumoto A 1979 Dependence of the electromechanical coupling coefficient on the width-to-thickness ratio of plank-shaped piezoelectric transducers used for electronically scanned ultrasound diagnostic systems J. Acoust. Soc. Am. 66 1609-11

[5] Naillon M, Coursant R H and Besnier F 1983 Analysis of piezoelectric structures by a finite element method Acta Electron. 25 341-62

[6] Lerch R 1990 Simulation of piezoelectric devices by twoand three-dimensional finite elements IEEE Trans. Ultrason. Ferroelectr. Freq. Control 37 233-47

[7] Guo N, Cawley P and Hitchings D 1992 The finite element analysis of the vibration characteristics of piezoelectric discs J. Sound Vib. 159 115-38

[8] Yong Y-K 1995 A new storage scheme for the Lanczos solution of large scale finite element models of piezoelectric resonators IEEE Ultrasonics Symp. pp 1633-6

[9] Yong Y-K and Cho Y 1994 Algorithms for eigenvalue problems in piezoelectric finite element analysis IEEE Ultrasonics Symp. pp 1057-62

[10] Ma Z-D, Kikuchi N and Cheng H-C 1995 Topological design for vibrating structures Comput. Methods Appl. Mech. Eng. 121 259-80

[11] Díaz A R and Kikuchi N 1992 Solutions to shape and topology eigenvalue optimization problems using a homogenization method Int. J. Numer. Methods Eng. 35 1487-502

[12] Yao Q and Bjorno L 1997 Broadband Tonpilz underwater acoustic transducers based on multimode optimization IEEE Trans. Ultrason. Ferroelectr. Freq. Control 44 1060-6

[13] Yang R J and Chuang C H 1994 Optimal topology design using linear programming Comput. Struct. 52 265-75

[14] Sigmund O 1996 On the design of compliant mechanisms using topology optimization Danish Center for Applied Mathematics and Mechanics Report 535

[15] Silva E C N, Fonseca J S O and Kikuchi N 1998 Optimal design of periodic piezocomposites Comput. Methods Appl. Mech. Eng. 159 49-77

[16] Hanson R and Hiebert K 1981 A sparse linear programming subprogram Sandia National Laboratories Technical Report SAND81-0297

[17] Bendsøe M P, Díaz A R, Lipton R and Taylor J E 1995 Optimal design of material properties and material distribution for multiple loading conditions Int. J. Numer. Methods Eng. 38 1149-70

[18] Meric R A and Saigal S 1991 Shape sensitivity analysis of piezoelectric structures by the adjoint variable method AIAA J. 29 1313-8

[19] Smith W A and Auld B A 1991 Modeling 1-3 composite piezoelectrics: thickness-mode oscillations IEEE Trans. Ultrason. Ferroelectr. Freq. Control 38 40-7

[20] Bandyopadhyay A, Panda R K, Janas V E, Agarwala M K, Danforth S C and Safari A 1997 Processing of piezocomposites by fused deposition technique $J$. Am. Ceram. Soc. 80 1366-72

[21] Haftka R T, Gürdal Z and Kamat M P 1990 Elements of Structural Optimization (Dordrecht: Kluwer) pp 276-77 